\title{
Generalização da integral de Fresnel
}

\author{
Joilson Ferreira de Carvalho (1) Paulo Sérgio Costa Lino
}

\section{Resumo}

$\mathrm{Na}$ literatura, existem várias classes e técnicas para calcular integrais impróprias, tais como a teoria dos resíduos, mudança de ordem de integração, transformadas de Laplace, etc. Neste artigo, iremos apresentar uma expressão analítica para a generalização de uma das integrais de Fresnel, para tal, usaremos as transformadas de Laplace mudança de ordem de integração e identidades envolvendo as funções beta e gama. Consideramos que o desenvolvimento de técnicas alternativas para calcular analiticamente as integrais impróprias é de suma importância para a compreensão da teoria e para o aprimoramento das técnicas atuais.

Palavras-chave: Integral de Fresnel; Função beta; Função gama.

\begin{abstract}
In the literature, there are several classes and techniques for computing improper integrals, such as residue theory, change of order of integration, Laplace transforms, etc. In this article, we will present an analytical expression for the generalization of one of the Fresnel integrals, for this, we will use Laplace transforms, change of order of integration and identities involving beta and gamma functions. We believe that the development of alternative techniques to Analytically calculating improper integrals is of paramount importance for the understanding of theory and for the improvement of current techniques.
\end{abstract}

Keywords: Fresnel Integral; beta function; Gamma function

\section{Introdução}

Em Cálculo, quando introduzimos a definição de integral definida, inicialmente trabalhamos com uma função $f$ definida em um intervalo limitado $[a, b]$, e consideramos que $f$ não tenha uma descontinuidade infinita nesse intervalo. No entanto, para os casos em que o intervalo é infinito ou $f$ possui uma descontinuidade infinita em $[a, b]$, a integral é chamada de imprópria [8]. Esse tipo de integral desempenha um grande papel em diversas áreas da Matemática e da Física.

Dentre as famosas integrais impróprias, destacamos as integrais de Fresnel [1, p. 83] definidas por:

$$
S(x)=\int_{0}^{x} \sin \left(t^{2}\right) d t
$$


e,

$$
C(x)=\int_{0}^{x} \cos \left(t^{2}\right) d t
$$

Elas foram desenvolvidas pelo engenheiro civil e físico francês Augustin Jean Fresnel (1788-1827), e originaram-se nos estudos da ótica, para explicar os fenômenos da difração da luz, como as chamadas franjas de interferências $[1]$.

As integrais de Fresnel também são usadas no método da fase estacionária, um instrumento de análise aproximada, usado em estudos de propagação ondulatória [2]. Além disso, elas compõem as equações paramétricas da Espiral de Cornur [1].

Neste artigo, iremos usar as transformadas de Laplace, função

Figura 1: Augustin Jean Fresnel Fonte: https://bit.ly/3FusCZO gama, função beta e mudança de ordem de integração para determinar uma expressão analítica para a generalização de uma das integrais de Fresnel (1), dada por

$$
I(a)=\int_{0}^{\infty} \sin \left(x^{a}\right) d x, \quad \text { com } \quad a>1
$$

Para isso, apresentaremos algumas definições e lemas preliminares que serão cruciais para obtermos $I(a)$.

\section{Definições e Lemas}

Nesta seção, expomos algumas definições e lemas que serão primordiais para obtermos o resultado desejado.

Definição 1. A função gama, denotada por $\Gamma(x)$, é definida pela integral imprópria

$$
\Gamma(x)=\int_{0}^{\infty} e^{-t} t^{x-1} d t
$$

com $x>0$, de modo que a integral convirja.

Definição 2. A função beta, denotada por $B(p, q)$ é definida por:

$$
B(p, q)=\int_{0}^{1} t^{p-1}(1-t)^{q-1} d t
$$

com $p>0$ e $q>0$.

Definição 3. Seja $f(t)$ uma função definida para $t>0$. A transformada de Laplace de $f(t)$, denotada por $F(s)$ é definida por

$$
\mathscr{L}\{f(t)\}=F(s)=\int_{0}^{\infty} e^{-s t} f(t) d t .
$$

Se a função $f$ é seccionalmente contínua (contínua por partes) e de ordem exponencial, então a transformada de Laplace $F(s)$ existe. Chama-se funções admissíveis, a classe de funções para os 
quais a transformada de Laplace existe. É possível verificar que se $f(t)=\operatorname{sen}(x)$, então $\mathscr{L}\{f(t)\}=$ $\frac{1}{1+s^{2}}$.

Lema 1. Seja $f:[0, \infty) \longrightarrow \mathbb{R}$ dada por $f(t)=t^{n}$. Se $n>-1$ e $s>0$, então

$$
\mathscr{L}\{f(t)\}=\frac{\Gamma(n+1)}{s^{n+1}}
$$

Demonstração. Fazendo $s t=u$, e supondo que $s>0$, temos

$$
\mathscr{L}\{f(t)\}=\int_{0}^{\infty} e^{-u}\left(\frac{u}{s}\right)^{n} d\left(\frac{u}{s}\right)=\frac{1}{s^{n+1}} \int_{0}^{\infty} u^{n} e^{-u} d u=\frac{\Gamma(n+1)}{s^{n+1}} .
$$

Do Lema (1) segue que, se $f(t)=t^{n-1}$, com $n \in \mathbb{N}^{*}$, temos

$$
\frac{1}{s^{n}}=\frac{\mathscr{L}\{f(t)\}}{\Gamma(n)}
$$

Lema 2. Se $p>0$ e $q>0$, então

$$
B(p, q)=\frac{\Gamma(p) \Gamma(q)}{\Gamma(p+q)}
$$

Demonstração. Considere,

$$
G(x)=\int_{0}^{x} t^{p-1}(x-t)^{q-1} d t
$$

Então, pelo teorema da convolução [8, p. 72 - 74], temos

$$
\mathscr{L}\{G(x)\}=\mathscr{L}\left\{x^{p-1}\right\} \mathscr{L}\left\{x^{q-1}\right\}
$$

Do Lema (1), segue que

$$
\mathscr{L}\{G(x)\}=\frac{\Gamma(p)}{s^{p}} \frac{\Gamma(q)}{s^{q}}=\frac{\Gamma(p) \Gamma(q)}{s^{p+q}} .
$$

Assim,

$$
G(x)=\mathscr{L}^{-1}\left\{\frac{\Gamma(p) \Gamma(q)}{s^{p+q}}\right\}=\frac{\Gamma(p) \Gamma(q)}{\Gamma(p+q)} x^{p+q-1} .
$$

Fazendo $x=1$, temos

$$
G(1)=\int_{0}^{1} t^{p-1}(1-t)^{(q-1)} d t=\frac{\Gamma(p) \Gamma(q)}{\Gamma(p+q)} 1^{p+q-1}
$$


Portanto,

$$
B(p, q)=\frac{\Gamma(p) \Gamma(q)}{\Gamma(p+q)} .
$$

Lema 3. Se $p>0$ e $q>0$, então

$$
\int_{0}^{\frac{\pi}{2}} \sin ^{2 p-1} \vartheta \cos ^{2 q-1} \vartheta d \vartheta=\frac{1}{2} \frac{\Gamma(p) \Gamma(q)}{\Gamma(p+q)}
$$

Demonstração. Do Lema (2), segue que

$$
B(p, q)=\int_{0}^{1} t^{p-1}(1-t)^{q-1} d t=\frac{\Gamma(p) \Gamma(q)}{\Gamma(p+q)} .
$$

Considerando $t=\sin ^{2} \vartheta$, temos

$$
\begin{aligned}
& \int_{0}^{\frac{\pi}{2}}\left(\sin ^{2} \vartheta\right)^{p-1} \vartheta\left(1-\sin ^{2} \vartheta\right)^{q-1} 2 \sin \vartheta \cos \vartheta d \vartheta=\frac{\Gamma(p) \Gamma(q)}{\Gamma(p+q)} \\
& 2 \int_{0}^{\frac{\pi}{2}} \sin ^{2 p-2} \vartheta \cos ^{2 q-2} \vartheta \sin \vartheta \cos \vartheta d \vartheta=\frac{\Gamma(p) \Gamma(q)}{\Gamma(p+q)}
\end{aligned}
$$

Da equação (17), segue que

$$
\int_{0}^{\frac{\pi}{2}} \sin ^{2 p-1} \vartheta \cos ^{2 q-1} \vartheta d \vartheta=\frac{1}{2} \frac{\Gamma(p) \Gamma(q)}{\Gamma(p+q)}
$$

Lema 4. Seja $x \in \mathbb{R}$ tal que $0<x<1$, então

$$
\Gamma(x) \Gamma(1-x)=\frac{\pi}{\sin (\pi x)}
$$

Demonstração. Veja em [8, p. 218 - 219].

Observe que, se $x=\frac{1}{2}$, então $\Gamma\left(\frac{1}{2}\right)=\sqrt{\pi}$.

\section{Resultado principal}

Usando a teoria dos resíduos [4, p. 231 - 233], obtemos

$$
I(2)=\int_{0}^{\infty} \sin \left(x^{2}\right) d x=\frac{1}{2} \sqrt{\frac{\pi}{2}}
$$


que representa a integral de Fresnel clássica. A desvantagem do uso dessa teoria é a escolha de um contorno adequado para realizar a integração complexa, cuja parte real irá fornecer a integral real [4, p. 206 - 235]. Outra técnica utilizada para resolver integrais impróprias é o uso de métodos clássicos baseados em transformadas de Laplace, função gama, função beta e mudança de ordem de integração. Para ilustrar essa técnica, iremos obter a expressão analítica de $I(\alpha)$ dada em (3), sendo um número real. É claro que se $a=2$, obtemos o resultado dado em (20).

Teorema 1. Se a> 1, a expressão analítica para a generalização da integral de Fresnel I(a) é dada por:

$$
I(a)=\frac{1}{a} \Gamma\left(\frac{1}{a}\right) \sin \left(\frac{\pi}{2 a}\right)
$$

Demonstração. Seja $u=x^{n}$, de modo que $d u=a x^{a-1} d x$. Mas $x=\sqrt[a]{u}$ então $d u=\frac{a u}{\sqrt[a]{u}} d x$ e $d x=\frac{\sqrt[a]{u}}{a u} d u$, assim

$$
I(a)=\int_{0}^{\infty} \sin (u) \frac{\sqrt[a]{u}}{a x} d u=\frac{1}{a} \int_{0}^{\infty} u^{\frac{1}{a}-1} \sin (u) d u=\frac{1}{a} \int_{0}^{\infty} \frac{1}{u^{1-\frac{1}{a}}} \sin (u) d u
$$

Da equação (6), segue que

$$
\frac{1}{u^{1-\frac{1}{a}}}=\frac{\mathscr{L}\{f(t)\}}{\Gamma\left(1-\frac{1}{a}\right)}=\frac{1}{\Gamma\left(1-\frac{1}{a}\right)} \int_{0}^{\infty} e^{-u t} t^{1-\frac{1}{a}+1} d t=\frac{1}{\Gamma\left(1-\frac{1}{a}\right)} \int_{0}^{\infty} e^{-u t} t^{-\frac{1}{a}} d t
$$

Substituindo a equação (23) na equação (22), temos

$$
I(a)=\frac{1}{a \Gamma\left(1-\frac{1}{a}\right)} \int_{0}^{\infty}\left(\int_{0}^{\infty} e^{-u t} t^{-\frac{1}{a}} d t\right) \sin (u) d u
$$

Aplicando a mudança de ordem de integração [5, p. 252] na expressão (25), segue que

$$
\begin{aligned}
I(\alpha) & =\frac{1}{a \Gamma\left(1-\frac{1}{a}\right)} \int_{0}^{\infty} t^{-\frac{1}{a}} \int_{0}^{\infty} e^{-u t} \sin (u) d u d t=\frac{1}{a \Gamma\left(1-\frac{1}{a}\right)} \int_{0}^{\infty} t^{-\frac{1}{a}} \mathscr{L}\{\sin (u)\} d t \\
& =\frac{1}{a \Gamma\left(1-\frac{1}{a}\right)} \int_{0}^{\infty} \frac{t^{-\frac{1}{a}}}{1+t^{2}} d t=\frac{1}{a \Gamma\left(1-\frac{1}{a}\right)} \int_{0}^{\infty} \frac{1}{t^{\frac{1}{a}}\left(1+t^{2}\right)} d t
\end{aligned}
$$

Seja $y=t^{\frac{1}{a}}$, de modo que $t=y^{a}$ e $d t=a y^{a-1} d y$. Assim,

$$
I(a)=\frac{1}{a \Gamma\left(1-\frac{1}{a}\right)} \int_{0}^{\infty} \frac{a y^{a-1}}{y\left(1+y^{2 \alpha}\right)} d y=\frac{1}{\Gamma\left(1-\frac{1}{a}\right)} \int_{0}^{\infty} \frac{y^{a-2}}{1+y^{2 \alpha}} d y
$$

Seja $y^{a}=\tan (\vartheta)$, de modo que $y=(\tan (\vartheta))^{\frac{1}{a}}$ e $d y=\frac{1}{a}(\tan (\vartheta))^{\frac{1}{a}-1} \sec ^{2}(\vartheta) d \vartheta$

Note que

$$
1+y^{2 a}=1+\tan ^{2}(\vartheta)=\sec ^{2}(\vartheta)
$$




$$
y^{a-2}=\left[(\tan (\vartheta))^{\frac{1}{a}}\right]^{a-2}=(\tan (\vartheta))^{1-\frac{2}{a}}
$$

Se $y=0$, então $\vartheta=0$, e se $y \longrightarrow \infty$, então $\vartheta=\frac{\pi}{2}$. Dessa forma,

$$
\begin{aligned}
I(a) & =\frac{1}{\Gamma\left(1-\frac{1}{a}\right)} \int_{0}^{\infty} \frac{(\tan (\vartheta))^{1-\frac{2}{a}}}{\sec ^{2}(\vartheta)} \frac{1}{a}(\tan (\vartheta))^{\frac{1}{a}-1} \sec ^{2}(\vartheta) d \vartheta \\
& =\frac{1}{a \Gamma\left(1-\frac{1}{a}\right)} \int_{0}^{\infty}(\tan (\vartheta))^{-\frac{1}{\alpha}} d \vartheta .
\end{aligned}
$$

Sendo $\tan (\vartheta)=\frac{\sin (\vartheta)}{\cos (\vartheta)}$, então

$$
I(\alpha)=\frac{1}{a \Gamma\left(1-\frac{1}{a}\right)} \int_{0}^{\infty}(\sin (\vartheta))^{-\frac{1}{a}}(\cos (\vartheta))^{\frac{1}{a}} d \vartheta
$$

Sejam $2 p-1=-\frac{1}{a}$ e $2 q-1=\frac{1}{a}$, então $p=\frac{1}{2}\left(1-\frac{1}{a}\right)$ e $q=\frac{1}{2}\left(1+\frac{1}{a}\right)$, assim

$$
I(a)=\frac{1}{a \Gamma\left(1-\frac{1}{a}\right)} \int_{0}^{\infty}(\sin (\vartheta))^{2 p-1}(\cos (\vartheta))^{2 q-1} d \vartheta
$$

Pelo lema (3),

$$
\int_{0}^{\infty}(\sin (\vartheta))^{2 p-1}(\cos (\vartheta))^{2 q-1} d \vartheta=\frac{1}{2} \frac{\Gamma\left(\frac{1}{2}-\frac{1}{2 a}\right) \Gamma\left(\frac{1}{2}+\frac{1}{2 a}\right)}{\Gamma\left(\frac{1}{2}-\frac{1}{2 a}+\frac{1}{2}-\frac{1}{2 a}\right)}=\frac{\Gamma\left(\frac{1}{2}-\frac{1}{2 a}\right) \Gamma\left(\frac{1}{2}+\frac{1}{2 a}\right)}{2}
$$

Substituindo a equação (32) na equação (31), temos

$$
\begin{aligned}
I(a) & =\frac{\Gamma\left(\frac{1}{2}-\frac{1}{2 a}\right) \Gamma\left(\frac{1}{2}+\frac{1}{2 a}\right)}{2 a \Gamma\left(1-\frac{1}{a}\right)} \\
& =\frac{\Gamma\left(\frac{1}{2}-\frac{1}{2 a}\right) \Gamma\left(1-\left(\frac{1}{2}-\frac{1}{2 a}\right)\right)}{2 a \Gamma\left(1-\frac{1}{a}\right)} .
\end{aligned}
$$

Pelo lema (4),

$$
\Gamma\left(\frac{1}{2}-\frac{1}{2 a}\right) \Gamma\left(1-\left(\frac{1}{2}-\frac{1}{2 a}\right)\right)=\frac{\pi}{\sin \left(\frac{\pi}{2}-\frac{\pi}{2 a}\right)} .
$$


Substituindo a equação (34) na equação (33), temos

$$
I(a)=\frac{\pi}{2 a \Gamma\left(1-\frac{1}{a}\right) \sin \left(\frac{\pi}{2}-\frac{\pi}{2 a}\right)}=\frac{\pi}{2 a \Gamma\left(1-\frac{1}{a}\right) \cos \left(\frac{\pi}{2 a}\right)} .
$$

Sendo $a>1$, então $0<\frac{1}{a}<1$, de modo que

$$
\Gamma\left(\frac{1}{a}\right) \Gamma\left(1-\frac{1}{a}\right)=\frac{\pi}{\sin \left(\frac{\pi}{a}\right)}
$$

assim,

$$
\frac{1}{\Gamma\left(1-\frac{1}{a}\right)}=\frac{\Gamma\left(\frac{1}{a}\right) \sin \left(\frac{\pi}{a}\right)}{\pi}=\frac{2}{\pi} \Gamma\left(\frac{1}{a}\right) \sin \left(\frac{\pi}{2 a}\right) \cos \left(\frac{\pi}{2 a}\right) .
$$

Substituindo a equação (37) na equação (35), temos

$$
I(a)=\frac{1}{a} \Gamma\left(\frac{1}{a}\right) \sin \left(\frac{\pi}{2 a}\right)
$$

Note que, se $a=2$, temos

$$
I(2)=\frac{1}{2} \Gamma\left(\frac{1}{2}\right) \sin \left(\frac{\pi}{4}\right)=\frac{1}{2} \sqrt{\pi} \frac{1}{\sqrt{2}}=\frac{1}{2} \sqrt{\frac{\pi}{2}} .
$$

\section{Considerações Finais}

As integrais impróprias desempenham um importante papel em diversas áreas da Matemática e da Física. Muitas das transformadas integrais são definidas através de integrais impróprias. Além disso, elas também estão presentes nas distribuições de probabilidades e nas definições de derivadas e integrais fracionárias [6].

Nesse sentido, desenvolver técnicas alternativas para calcular analiticamente as integrais impróprias é de fundamental importância para a compreensão da teoria e para o aprimoramento das técnicas atuais. Como trabalho futuro, desejamos usar as técnicas apresentadas nesse artigo para resolver outras integrais impróprias da Física-Matemática.

\section{Referências}

[1] ÁVILA, Geraldo.Contatos de Curvas, Círculo Osculador e Integrais de Fresnel, Matemática Universitária, № 2, 1985, p. 75-85.

[2] ÁvILA, Geraldo.O Cálculo das Integrais de Fresnel, Matemática Universitária, № 5, 1987, p. $77-81$.

[3] ÁVILA, Geraldo.O Método da Fase Estacionária, Matemática Universitária, № 9/10, 1989, p. 134-143. 
[4] ABLOWITZ, M. J.; FOKAS, A. S.Complex Variables: Introduction and Applications, Cambridge, Cambridge University press, 1997.

[5] BARTLE, Robert G. Elementos de análise real, Rio de Janeiro, 1983.

[6] CARMO, Rubens de Figueredo; OLIVEIRA, Edmundo Capelas de. Cálculo Fracionário, Livraria da Física, 2015.

[7] STEWART, James. Cálculo, 7ª edição, Volume I, São Paulo, Cengage Learning, 2013.

[8] SPIEGEL, Murray R.Transformadas de Laplace, São Paulo, Mc Graw-Hill, 2003.

Joilson Ferreira de Carvalho Universidade do Estado de Mato Grosso (Unemat) $<$ joilson.carvalho@unemat.br>

Paulo Sérgio Costa Lino Universidade do Estado de Mato Grosso (Unemat) $<$ linnux2001@gmail.com>

Recebido: $02 / 06 / 2020$

Publicado: $14 / 02 / 2022$ 